\title{
Análisis complejo del discurso
}

Javier Alberto Piloto Rodríguez (Cuba)

Instituto de Filosofía de la Academia de Ciencias de Cuba javier@filosofia.cu
Complex Discourse Analysis

\author{
Olga Rosa González Martín (Cuba) \\ Asociación Cubana de Comunicadores \\ socialesolgarosa@cehseu.uh.cu
}

\section{Resumen \\ El artículo se propone llevar el estudio del discurso al cambio de paradigma que se ha producido en las últimas décadas en las ciencias sociales. Propone un análisis de discurso de nuevo tipo desde la teoría de la complejidad. A partir del estudio bibliográfico documental, hace una crítica que incorpora nuevos elementos del pensamiento científico de diferentes campos de estudio y se efectúa el respectivo cambio de noción en la concepción tanto del objeto de}

\section{Abstract}

This article studies the paradigm change that has occurred in social sciences during the last decades. It also suggests a new discourse analysis from the theory to the complexity. From a bibliographic study, it incorporates new elements of the scientific thought of different fields of study and changes the idea of the object of study conception as well as its approach. Although this study is not finished, it explores a territory that needs to be deeply systematized. For this reason, 
estudio como de la forma de abordarlo. No obstante, no constituye un estudio acabado, sino una exploración en un terreno que necesita ser sistematizado con mayor profundidad. De este modo, lo que hemos dado en llamar análisis complejo del discurso es una invitación inicial a un campo que recién abre sus puertas.

Palabras clave: análisis del discurso, análisis crítico del discurso, teoría de la complejidad, análisis complejo del discurso. this is an invitation to explore a field that just opened its doors.

Keywords: Discourse analysis, critic discourse analysis, complexity theory, complex discourse analysis. 


\section{Análisis complejo del discurso}

Complex Discourse Analysis

\author{
Javier Alberto Piloto Rodríguez (Cuba) \\ Periodista por la Universidad de La Habana. Cursa \\ la Maestría en Relaciones Internacionales del \\ Instituto Superior de Relaciones Internacionales \\ de Cuba. Investigador del Instituto de \\ Filosofía de la Academia de Ciencias de Cuba. \\ Correo electrónico: javier@filosofia.cu \\ Especialista en Periodismo de investigación \\ Instituto de Filosofía del Ministerio de \\ Ciencia, Tecnología y Medio Ambiente
}

Olga Rosa González Martín (Cuba)

Miembro del Círculo de Investigadores de la Asociación Cubana de Comunicadores Sociales, de la Asociación Cubana de las Naciones Unidas y de la Asociación de Estudios Latinoamericanos. Correo electrónico:

Correo electrónico: olgarosa@cehseu.uh.cu Doctora en Ciencias de la Comunicación

Centro de Estudios Hemisféricos y sobre los Estados Unidos de la Universidad de La Habana

\section{Introducción}

El análisis del discurso es ampliamente demandado hoy por los más variados actores sociales. Desde los políticos hasta los líderes comunitarios, pasando por la academia, reclaman su utilización tanto teórica como práctica para optimizar sus gestiones en el proceso comunicativo. Innumerables son los autores que se han especializado en esta temática y producido extensa bibliografía, tales como Van Dijk (1996, 2005), Thompson (2008), entre otros. Todos ellos enmarcados en el paradigma del racionalismo clásico, al cual sigue aferrada casi la totalidad de la academia cubana.

Aún en pleno siglo XXI las más modernas investigaciones de este tipo desconocen totalmente los avances más recientes salidos del paradigma de la complejidad (Morin, 1992, 1990). Con ello, se desecha todo un potencial científico de última generación capaz de llevar nuestras investigaciones a un nivel cualitativamente superior. 
Ante el reto de implementar estos saberes, a fin de realizar un análisis de discurso de nuevo tipo, proponemos una nueva manera de hacer análisis del discurso desde la teoría de la complejidad. Hay que resaltar que no constituye un estudio acabado, sino una exploración en un terreno que necesita ser sistematizado con mayor profundidad. De este modo, lo que hemos dado en llamar análisis complejo del discurso es una invitación inicial a un campo que recién abre sus puertas.

Para introducir esta propuesta partiremos fundamentalmente de las tesis de Teun van Dijk como núcleo de las críticas en las que se fundamenta este trabajo, y luego (a modo de antítesis), se irán incorporando elementos tomados de las teorías de la complejidad hasta llegar (como síntesis) al análisis complejo del discurso.

\section{Marco teórico}

Van Dijk (1996, p. 69) considera que el discurso, ya sea oral, ya sea escrito, se define como un acto comunicativo de un tipo especial, estrechamente relacionado con actividades comunicativas no verbales y semióticas de significado y con los usos sociales de códigos simbólicos, como los de la comunicación visual. Por otra parte, para el filósofo ruso Todorov (1990) el discurso es la fijación en las estructuras mentales, a diferencia de un texto "material". El discurso, entonces, pertenece al acto de enunciación (verbalización del sentido) y el texto es la enunciación (la "fictización” del texto) (Timoschuk, 2010).

En el discurso político, es lo que concierne a los actores políticos (Van Dijk, 1994). Sin embargo, desde un punto de vista del entorno comunicativo, plantea que sería necesario incluir a los receptores de este proceso comunicativo político. En este sentido, Golovina (2008) afirma que el discurso político es una compleja y multidimensional construcción, que incluye no solo a las instituciones, sino a las relaciones personales $y$ que posee una determinada composición de géneros, los cuales pueden ser clasificados según los niveles de institucionalización, diferenciación, relaciones sujeto-objeto, los socioculturales y los sucesos.

Según Howarth (s. f., p. 124), la teoría del discurso se interesa por el papel que representan las prácticas e ideas sociales significativas en la vida política. Analiza de qué manera los discursos configuran la comprensión que las personas tienen de sus propios 
papeles sociales y cómo influyen en sus actividades políticas.

En la literatura consultada al respecto, salta a la vista un primer bloque por la importancia epistemológica y organizativa de las categorías llevadas a estudio: el análisis del discurso ideológico. Van Dijk (1996, p. 15) considera que su propuesta resulta pertinente debido a que "las personas generalmente adquieren, expresan y reproducen sus ideologías por medio del texto o del habla", con la función de "poner al descubierto la ideología de hablantes y escritores a través de una lectura minuciosa”. Así, define la ideología como "los sistemas que sustentan las cogniciones sociopolíticas de los grupos" (p. 18).

En los estudios de este primer bloque que examinamos, es muy importante el papel que se le otorga a la relación entre la estructura del discurso y la estructura social. Aquí se demanda la necesidad de descubrir cómo se articulan ambas. Sin embargo, antes de llegar a este aspecto, definamos otros conceptos importantes.

El eje fundamental de la propuesta teórica de Van Dijk es la interrelación entre los elementos que conforman el triángulo cogniciónsociedad-discurso (Egüez, 1999). En esta pirámide, las bases serían la sociedad y el discurso; y la cima, o sea, el más importante de los mediadores, la cognición, a la que le atribuye un papel determinante. En esencia el análisis del discurso se encarga de revelar la incidencia de los contextos cognitivos, sociales, culturales, etc., en los contextos, significados, estructuras y estrategias del texto.

En el segundo bloque del análisis del discurso, se encuentra el análisis crítico del discurso. Esta propuesta se detiene fundamentalmente en los conceptos de poder, dominación y hegemonía, así como en los contextos, las situaciones comunicativas, los modelos mentales y la manipulación ideológica. Desde esta perspectiva, y según consideraciones de Santander (2011), el discurso es entendido como una práctica social, y desde esa convicción, inicia y justifica sus análisis discursivos como análisis sociales.

Aquí la función del analista del discurso sería la de examinar

de qué modo las estructuras de significado hacen posibles ciertas formas de conducta. Al hacer esto, pretende comprender cómo se generan los discursos que estructuran las actividades de los agentes sociales, cómo funcionan y cómo se cambian. Al empeñarse en entender estos 
objetos de investigación el analista del discurso da prioridad a conceptos políticos como "antagonismo", "actuación", "poder", y "hegemonía” (Howarth, s. f., p. 125).

Entre los diferentes tipos de análisis del discurso que existen, Van Dijk (1999, p. 10) habla también sobre el análisis del discurso político, el cual

trata tanto sobre el discurso político a la vez que es una empresa crítica. En el espíritu de los enfoques contemporáneos respecto del ACD [análisis complejo del discurso] esto significaría que el análisis de discurso crítico-político consiste especialmente en el estudio de las formas de reproducción del poder político, la dominación o el abuso de poder mediante el discurso político, incluyendo las diversas formas de resistencia o las muestras de poder contra tales formas de predominio discursivo.

"El análisis del discurso político, en muchos aspectos, será como el de cualquier otro tipo. Lo específico debería buscarse en las relaciones entre las estructuras de discurso y las estructuras contextuales políticas" (Van Dijk y Mendizábal, 1999, p. 37). Estos dos estudiosos señalan además que no podemos identificar el análisis del discurso político con un análisis político en cuanto el primero será usado por los politólogos o políticos en caso de reportarles un beneficio a sus trabajos.

La principal carencia es la ausencia de un método fijo para el análisis (Van Dijk, 1996, 2004, 2005; Santander, 2011), pues existen tantos métodos como autores. El resto de las fisuras han sido enumeradas por el propio Van Dijk a partir de las siguientes interrogantes:

¿Cómo poner al descubierto la ideología de los comunicantes? ¿Cómo se relacionan las estructuras del discurso con las estructuras sociales? ¿Qué estructuras se articulan entre discurso e ideología? ¿Qué factores mentales influyen en la producción del discurso?

Por otra parte, Van Dijk insiste en varias de sus producciones en que es necesario desarrollar nuevas teorías y métodos de investigación, más ricos y complejos que los existentes. Por ello, nosotros optamos por la propuesta de un análisis complejo del discurso.

Según Howarth los presupuestos filosóficos de la teoría del discurso han recibido dos acusaciones principales. 
La primera le acusa de ser idealista y la segunda de ser una variante del relativismo. Respecto a la primera, ciertos críticos "realistas" afirman que la categoría de discurso lo reduce todo a pensamiento o a lenguaje. Se considera "idealismo", en su sentido más amplio, el reducir la realidad a las ideas o conceptos que nosotros tenemos de ella. Por el contrario, se considera "realismo" al hecho de que haya una realidad independiente de dichas ideas o conceptos (Howarth, s. f., p. 136).

Por otra parte, en cuanto a las teorías sustantivas, las críticas a la teoría del discurso señalan

tres problemas importantes: 1) la completa fragmentación e inestabilidad de las estructuras y relaciones sociales; 2) el abandono del concepto de ideología y el deterioro de sus puntos de apoyo críticos, y 3) la incapacidad (o falta de voluntad) para analizar las instituciones sociales o políticas (Howarth, s. f., p. 138).

Para Van Dijk los discursos son una parte estructural de sus contextos y sus estructuras respectivas se influyen mutua y continuamente. ¿Cómo? ¿Él responde esta cuestión?
Afirma que no es una relación directa, sino que está mediada por la cognición social y las estrategias de producción o comprensión del discurso. De aquí se entiende la importancia que él les atribuye a los modelos mentales (representaciones concretas de hechos específicos) y los procesos del conocimiento.

Según lo que nos explica Van Dijk, el discurso es el mediador entre el sujeto y el mundo al que pertenece. Es quien posibilita la puesta en común de ambas realidades y su comprensión y aprehensión mutuas. ¿Qué naturaleza le podemos otorgar a este discurso: dependiente $o$ independiente de los actores que relaciona? ¿Existe la retroalimentación en este proceso? ¿Pudiéramos encontrar puntos significativos de inflexión en su funcionamiento? ¿Qué tipo de estructura presenta este sistema en todo su conjunto?

En parte respondiendo a este último interrogante, podemos ver que las estructuras que presenta Van Dijk son de un discurso-texto. Él no ofrece una estructura general, común a todos los tipos de discursos. O sea, no ofrece una estructura del discurso en tanto tal. ¿Y de qué nos serviría esto? Lograr modelar el funcionamiento de un discurso como sistema, es decir, 
en su estado abstracto más puro, pudiera arrojar los mejores resultados posibles en cuanto a neutralidad y desprejuicio se refiere.

Por otra parte, si retomamos el planteamiento inicial, referente a la esencia del análisis, pudiéramos partir de Bushev (2013) cuando planteó que el análisis del discurso de origen político actual es realmente complejo, y que la metodología de dicho análisis solo se elabora por sí misma. De ahí que este sea nuestro punto de partida para la propuesta que hacemos.

\section{Metodología}

Para descubrir cuál es la forma de la estructura de un discurso, partiremos de la metodología que nace del modelo de simulación basado en agentes. Las ciencias sociales han estado siempre entre dos modos de hacer ciencia, diversos y enfrentados entre sí. Por un lado, tenemos a los defensores del método hipotético deductivo que postulan la necesidad de partir de una serie de reglas a priori y deducir a partir de estas la existencia de patrones de conductas. Por otro lado, nos encontramos con los defensores del método inductivo, quienes proponen que ante la falta de un conocimiento de primera mano del objeto de estudio es necesario la recolección de datos que permita establecer generalizaciones empíricas a partir de la cual se conoce nuestro objeto de estudio (Miceli et al., 2005).

Sin embargo, los modelos basados en agentes toman un camino intermedio, una tercera vía. Se trata de una construcción del investigador, o sea, este elabora un modelo que le permite establecer a priori las reglas del proceso por investigar, con lo cual se acerca al método deductivo, aunque se diferencian en cuanto un modelo no equivale a la verdad, sino que se trata de un pronóstico. A su vez, esto los acerca a los modelos inductivos porque se transforma en una muestra más aplicable al objeto de estudio (Miceli et al., 2005).

Esta modelación, síntesis de todas las conclusiones abordadas hasta el momento, se inspira para su funcionamiento "concreto" en los principios metodológicos del modelo de simulación basado en agentes y la dinámica de sistemas. En lo metodológico, si partimos del modelo de simulación basado en agentes, debemos tener presente que el modelo resultante en esta primera instancia será solo un esbozo inacabado porque, según la propia definición de esta metodología, la recolección de datos 
empíricos es determinante para su confección. Por otra parte, en técnicas y métodos concretos, tomaremos por separado lo que nos interesa de cada uno de estos modelos, en específico los mencionados arriba.

El primero nos exige definir los agentes presentes en el sistema, su carácter (nivel de independencia), metas y objetivos individuales, las reglas de conducta entre los agentes y entre estos y el entorno, la representación explícita e individual de los agentes, la representación explícita de las redes de interacción, el análisis de atributos y comportamiento de los individuos y los procesos de adaptación y procesos de emergencia.

Paralelamente, la dinámica de sistemas como técnica nos permitirá, en primer lugar, identificar las variables y las relaciones entre ellas, prestar atención a la retroalimentación (tanto positiva como negativa), y a partir de ahí la obtención de causas y efectos entre las partes del sistema; así comprenderemos las causas estructurales que provocan el comportamiento del sistema, podremos identificar las relaciones de flujo y acumulación (stocks), así como las no linealidades, y encontraremos pruebas de políticas alternativas en el modelo en cuestión. Contrariamente a estos beneficios, está el carácter secundario que presta a los datos históricos, algo que en nuestro caso deberá tener relevancia primaria, y que lograremos solventar con el modelo de simulación basado en agentes. Si en lo teórico pudieran parecer incompatibles y hasta contradictorios entre sí, a los fines prácticos de nuestra investigación son evidentemente complementarios, cada uno otorga algo diferente y juntos completan el constructo del que prescindimos para guiar la investigación. Solo nos queda como reto saber conjugarlos oportunamente y no obtener resultados que lejos de dar luces solo sirvan para alejar a la investigación del camino de la ciencia. Este tipo de detalles se explicitarán más adelante.

Empecemos, entonces, con el mapa que integrará este modelo mixto.

\section{Constifuyentes del sistema Agentes}

Comunicantes (C): emisores-receptores que intervienen en el juego. En el modelo por desarrollar, solo tendremos a $\mathrm{C} 1$ y C2, el mínimo que podamos encontrar. Respecto de su carácter, el nivel de independencia de estos quedará al descubierto en el proceso de observación; por el momento podemos plantear a manera 
de hipótesis que su comportamiento fluctúa entre la independencia total y la observación mutua para la toma de decisiones. Lo mismo se aplica en sus metas y objetivos.

\section{Elementos}

Reglas de conducta entre agentes: las reglas del juego se basan en el deseo de cada jugador en obtener la mayor cantidad de influencia sobre el otro. Dicha influencia puede fluctuar desde una simple invitación a imitar su conducta hasta la voluntad de querer controlar totalmente el proceder del otro.

Reglas de conducta agente-entorno: van encaminadas a la obtención de reconocimiento y de la mayor cantidad de primas que ingresen desde fuera, así como a emitir al exterior la producción más influyente. El entorno siempre querrá influir sobre los agentes.

Representación explícita: $\mathrm{C} 1$ y C2.

\section{Variables presentes en el sistema}

Intención (I): el deber-ser de lo que el comunicante desea poner en juego.

Texto (T): conjunto de símbolos (escritos, visuales, directos, indirectos, perceptibles, imperceptibles, conscientes, inconscientes...) que componen lo que el comunicante efectivamente pone en juego.

Impacto (P): impresión que el comunicante 1 tiene de los "resultados" de su texto en el comunicante 2. Esto no quiere decir que así sea, es solo su impresión.

Contratexto (O): respuesta al texto $^{1}$.

Como es evidente, todas ellas tienen un carácter dependiente al comunicante que las introduce en el juego. En ellas están presentes los llamados círculos causales.

Redes de interacción (RI): explicitan las conexiones entre las variables y entre los agentes y las variables. Una mayor precisión se encontrará más adelante cuando desarrollemos el contenido del análisis de este modelo. Análisis de atributos (AA): dentro de las redes de interacción son los que nos darán la medida de lo que puede aportar cada una de las variables al proceso de interacción, dado el sello que le "imprimen" los mismos agentes.

1 Para analizar estas dos últimas variables, se puede acudir a otra que integra a ambas y que denominaremos "replicación", dado lo complicado que se puede volver para una investigación lograr incluir el "impacto" en su totalidad, porque requeriría de varios estudios complementarios y todo un equipo de investigadores para su solución. Esta variable resume las funciones $P$ y $O$. 
Retroalimentación (R): es la apropiación y reconversión de la respuesta de $\mathrm{C} 2$ por parte de $\mathrm{C} 1$. Sirve de sustancia para el nuevo ciclo. Dentro de esta podemos encontrar la retroalimentación negativa (RN) y la retroalimentación positiva (RP). La primera está encargada de eliminar las anomalías y productos e informaciones que dañen a los agentes de forma individual y al sistema de forma general. La segunda permite que avancen los procesos destructivos en el sistema.

Aquí colocaremos, aunque no estén directamente relacionadas:

Las relaciones de stock (RS): se presentará como el cúmulo de información y poder informativo con que cuente el agente. Serán sus reservas y capacidad de acción para enfrentar al otro comunicante.

Las relaciones de flujo (RF): son las que posibilitarán (o impedirán) la acumulación de stocks en los agentes.

Metacontexto (M): es la longitud total de la línea consecutiva en la situación discursiva. Mide el grado de complejidad que esta alcanza.
Puntos de bifurcación (B): lugar donde se produce un cambio(s) en la dirección del discurso.

\section{Elementos del entorno}

Contexto: determina la longitud de las líneas que unen los puntos en el primer círculo de la "tela de araña". Dentro de este, podemos encontrar la estructura social —entorno social con el que se articula la estructura discursiva- y el ámbito internacional - estructura internacional, entorno mayor inmediato al entorno nacional donde se encuentra la estructura social que nos interesa-. No obstante, todo siempre dependerá del caso concreto de estudio.

Tiempo: es el promedio de la suma de las distancias que hay desde los puntos externos al atractor extraño ubicado en el centro de la figura, o sea, el fin de la situación discursiva.

Así, vistos todos los elementos, podemos presentar una imagen que grafica el modelo de dos comunicantes (figura 1): 


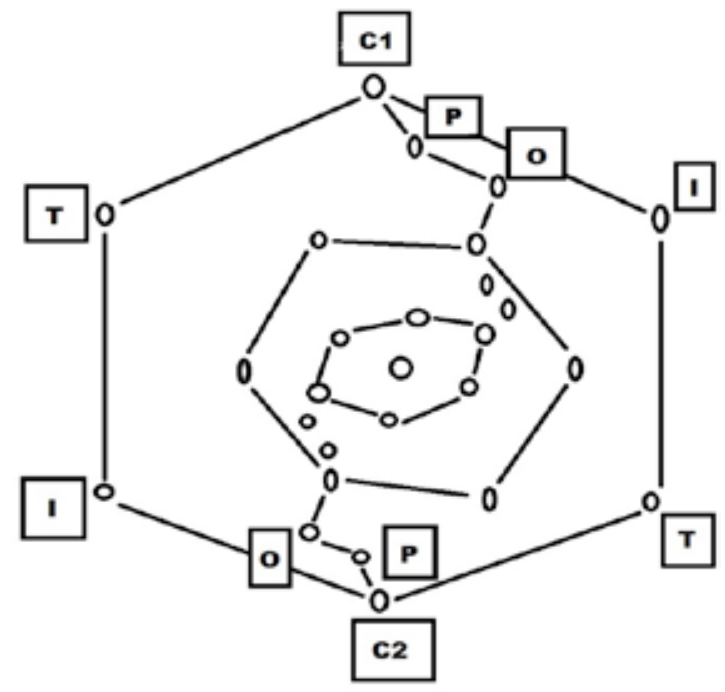

Figura 1. Modelo de 2 comunicantes (diagrama de influencia).

Esta figura podría describirse como una vista superior de un torbellino, formado por círculos delimitados por los puntos que representan a los agentes y variables presentes en la situación discursiva. El punto central es el atractor extraño alrededor del cual se plantea el juego ${ }^{2}$. Las líneas son las redes de interacción que conectan a los agentes con las variables que ponen en juego, proceso en el que también está comprendida la retroalimentación. Aquí están presentes también las relaciones de flujo y stocks.

De este modo, entenderemos como discurso al producto de las

2 Visto en el sentido de la teoría del juego (Morgenstern y Neumann, 1947). interacciones adaptativas y emergentes que se dan entre los agentes que intervienen en una situación discursiva. Su dinámica viene dada por el grado de complejidad de estos agentes (internos y externos) y sus interacciones. Su estructura interna se rige por el principio de autosimilaridad y no es más que la reproducción de reflejos de los agentes y las interacciones que le dan origen. En la figura 1 sería la línea espiral continua que une los puntos de bifurcación hacia donde se desplazan los comunicantes cuando pasan de un círculo a otro.

La situación discursiva sería el espacio propicio para la creación de un discurso. Su origen puede ser premeditado o azaroso. Está compuesto 
por los agentes y sus variables. Este sistema comunicativo abierto siempre se va a encontrar en un contexto y va a recibir su influjo. Toda situación discursiva tenderá a un atractor extraño, cuya función principal será la realización plena del discurso (se presenta como su fin).

\section{Resultados}

Una vez enunciados los elementos que conforman nuestro modelo, estamos en condiciones de explicitar en qué consiste nuestro tipo de análisis.

Para el análisis complejo del discurso, el comunicante (tal vez "usuario del discurso" para Van Dijk) no puede controlar su propia estrategia discursiva, ni siquiera está totalmente consciente de su propio texto e impacto. Las estrategias se desarrollan a través de principios caóticos (de la teoría del caos) y otros que se acercan a elementos planteados por la teoría de las catástrofes. En el modelo expuesto, se representaría a través de los cambios de dirección que se producen en cada punto de bifurcación.

Una de las concepciones que todavía arrastra el análisis del discurso es la de "hablantes" (emisores) y "receptores", que limita el potencial comunicativo de los sujetos presentes en un acto de comunicación. El análisis complejo del discurso, al incorporar la noción de comunicante, reconoce a plenitud las aptitudes presentes en un agente de determinada situación discursiva, donde más que sumar las propiedades del emisor y el receptor, presta atención a las propiedades emergentes que resultan de dicha síntesis.

Es importante aclarar que en este trabajo, de aquí en adelante, cuando se utilice el término discurso, se ceñirá estrictamente al concepto salido del modelo planteado por el análisis complejo del discurso (lo señalaremos al escribirlo con letra inicial mayúscula). Esto elimina la dualidad semántica que existe, por ejemplo, con el del análisis del discurso, cuando puede ser una categoría de análisis general o como sinónimo de conversación, declaración, texto específico, etc. Aquí solo se denominará discurso al resultado de una situación discursiva (posible solamente con al menos dos comunicantes). Es importante señalar que un discurso no puede ser logrado o emitido por un comunicante por sí solo; un discurso es resultado de las propuestas, intenciones y retroalimentaciones de las acciones que se plantean al menos dos comunicantes en una situación discursiva. 
Tal vez lo que el análisis del discurso entiende por "discurso" para el análisis complejo del discurso sería el equivalente del tramo de la situación discursiva "C1-I-T-C2-P", lo que recibe el nombre de intención discursiva. Esta diferencia es importante remarcarla porque rescata una noción de discurso que se acerca más a su verdadera complejidad. Para el análisis complejo del discurso, la idea de discurso no se permite el descuido de los elementos de la inconsciencia (que expone, digamos, el psicoanálisis), del azar y del caos, por lo que se puede afirmar que un discurso está por encima de lo que un simple comunicante desea transmitir o que efectivamente transmite; e incluso por encima de las pretensiones de los comunicantes presentes en el juego. Un discurso es un resultado, un discurso es cada una de las partes puede sacar de él supuestos "beneficios" o "daños", pero difícilmente podría tener la certeza total de esto más allá del corto plazo. Un discurso es un SCA, si se quiere, incluso, una superestructura, con propiedades emergentes.

En este trabajo nunca se hablará del discurso de $\mathrm{X}$ o Y comunicante, en todo caso, de la "intención comunicativa o discursiva" de uno de estos (también pudiera darse el caso de que se le denomine como "principios", "sistema de ideas"; por ejemplo: los principios del liberalismo, las ideas del socialismo, etc.). Para referirse aquí a discurso, solo se hará en los términos de "discurso de X/Y comunicantes", por poner un ejemplo. El discurso se va más allá de las manos de los propios agentes que le dieron origen (emergencia, y si se quiere incluso autopoiésis).

Van Dijk le presta mucha atención al discurso en calidad de utilización del lenguaje. Con ello, pretende ver sus dimensiones de comunicación e interacción. Para el análisis complejo del discurso detenerse en tales cuestiones supondría la creación de una "subdisciplina", porque dar prioridad a solo una de las aristas que intervienen en la situación discursiva sería optar por los principios de la reducción y la linealidad, lo que entraría en plena contradicción con lo concluido hasta aquí. Analizar de forma compleja un discurso significa tener en cuenta al todo y a cada una de sus partes al mismo tiempo.

Van Dijk confiesa y alerta el carácter inexplorado del lenguaje no verbal y la importancia que este posee en el acompañamiento del verbal (ver en especial Van Dijk, s. f., pp. 27-30). Para el análisis complejo 
del discurso es imposible desligar los lenguajes verbal y no verbal en el estudio de las variables texto e impacto (además de contratexto). Si se quiere obtener un estudio serio al respecto, lo pertinente será desarrollar ambos al mismo tiempo en una articulación indisoluble. Tal vez quedaría como un reto para el análisis complejo del discurso (posiblemente solucionado luego de la puesta en práctica del experimento en el estudio de caso) demostrar si el "análisis del discurso en sî" es capaz de desmantelar por sí solo esas incógnitas que el análisis del discurso ideológico pretende develar, por poner un ejemplo.

Van Dijk (s. f., p. 31) plantea: “Un aspecto crucial de muchas descripciones del discurso es el nivel del sentido, tal como lo analiza típicamente la semántica". En el análisis complejo del discurso, esto entraría fundamentalmente dentro del estudio del texto, el impacto, el contratexto y la retroalimentación.

Mas debemos ir más allá de la concepción que el análisis del discurso tiene sobre los participantes del discurso, sobre todo de aquellos que pretenden ejercer la dominación sobre los demás, y comprender el acto de creación del discurso como un juego competitivo entre los comunicantes que intervienen en él, donde pequeños cambios producen grandes resultados y que con muy poco y en desventaja se puede hacer mucho. Tenemos que desprendernos de la lógica determinista y lineal anterior que nos priva de comprender las potencialidades del discurso resultante de todo juego (situación discursiva). Debemos empezar a ver el discurso como un sistema complejo adaptativo con propiedades emergentes y que solo estudiándolas a fondo nos permitirá tener conciencia de su lógica sistémica.

Es importante destacar que cuando más adelante hablemos de "discurso político" o cualquier otro no nos encontramos ante contradicción alguna con los postulados del análisis complejo del discurso. Así como existen diferentes tipos de situaciones discursivas (familiares, educacionales, políticas), también vamos a encontrar discursos familiares, educativos, políticos, etc. Si en una situación discursiva familiar los temas tratados son de temática familiar, evidentemente el discurso resultante será familiar. Si dicha situación discursiva aborda temas económicos, estamos en presencia de un discurso económico-familiar. Y es aquí cuando el contexto, la temática y la situación discursiva en conjunto 
delatan la primera esencia compleja del discurso: su origen. Siempre lo primero que debemos identificar sobre un discurso es su origen: ¿quién?, ¿dónde?, ¿cuándo?, ¿qué se trató (temáticas y derivadas)? A partir de aquí se pasaría al segundo nivel: ¿cómo?, ¿para qué?, ¿por qué?

Si se partiera de la idea de que el análisis complejo del discurso es más una teoría general sobre el discurso, ¿cómo cabe entonces la pretensión del estudio de un caso concreto, específico? Lo primero aquí sería revisar lo que se entiende por general; $y$ no sería más que aquello que incluye y comprende todo. Si lo tomamos así no habría contradicción, sino una carencia de instrumentos y métodos para analizar lo particular. ¿Sería entonces necesario elaborar tomando como referencia al análisis complejo del discurso otros tipos de análisis para estudios más específicos? ¿Cuáles serían? ¿Cómo serían? Tendríamos que partir de las demandas básicas que se imponen: se trata de un discurso político, de un momento histórico dado, según el problema de investigación y los objetivos específicos, y lo que nos interesa es analizar su dinámica. A partir del paso intermedio que constituyó la elaboración del modelo intermedio o abstracto "modelo de dos comunicantes" expuesto anteriormente, es hora de pasar a la construcción del modelo formal.

\section{El modelo formal}

Este modelo tiene como tarea esbozar directamente la estructura del sistema que se desea analizar, es decir, el discurso en cuanto tal. Si bien el modelo intermedio fundamentó el medio de donde nace el discurso, o sea, la situación discursiva, así como todos los elementos, reglas y lógicas principales, la misión de este otro modelo nos lleva en concreto al objeto de estudio. Naturalmente, para poder llegar hasta aquí fue necesario tomar un estudio de caso para completar la fundamentación teórica correspondiente. A continuación, se enumeran $\mathrm{y}$ definen los elementos que forman parte de la estructura del discurso:

1. Idea (más argumentos, más secuencias lógicas): unidad básica de los flujos comunicativo-informacionales que se desprenden de la situación discursiva y fluyen a través de las estructuras del discurso y le dan sentido a su devenir.

2. Flujos de ideas: corrientes de ideas a través de la estructuras del discurso. Son las encargadas 
de dotar al sistema de integración y energía para su movimiento. Interconectan a la mayoría de los componentes de la estructura del discurso.

3. Banco de ideas: reservas acumulativas del sistema. Su utilización consiste en conservar reservas de ideas y sus derivados para implementarlas y servirse de ellas en un momento posterior al que fueron creadas y guardadas.

4. Reglas de desenvolvimiento: creadas dentro de la propia dinámica del sistema con el transcurrir del tiempo. No tienen nada que ver con las reglas de comportamiento entre los agentes o entre estos y las variables. Describe el comportamiento del modelo formal.

5. Zonas de rechazo: sectores restringidos y rechazados por el discurso a partir de las propias lógicas que lo constituyen. Influyen en la trayectoria de la línea recorrida por el discurso en el mapa trazado en la situación discursiva.

6. Exclusas: barrera que impide el acceso a determinadas zonas (se incluyen las de rechazo), la salida del sistema y la entrada de elementos negativos o dañinos. Son una especie de compuerta guardiana del sistema.

7. Defensas: encargadas del paso del discurso a un nuevo estadio, dígase después de cada punto de bifurcación. También pueden encontrarse dentro de los procesos autopoiéticos del sistema.

8. Flujos alternativos: flujos paralelos, auxiliares, complementarios o contrarios del flujo principal del discurso.

9. Zonas de indeterminación: resultado directo en que determinado segmento de la situación discursiva esté dañada, es decir, la pérdida de propiedades y eficiencia de, al menos, uno de los agentes provoca el incumplimiento de las reglas de conducta entre estos y entre estos y las variables, lo que provoca indeterminación en la comprensión de resultados. Dentro de la estructura propia del discurso se traduce en huecos, incoherencias y borrosidad en los elementos.

10. Recicladores: reprocesan unidades básicas y secundarias para reutilizarlas en otro momento del discurso.

11. Impulsores: aceleran la actividad de las unidades básicas y 
aumentan la eficiencia de su gestión.

12. Activadores: son los encargados de despertar unidades básicas que se encuentran en los bancos de ideas y sacarlas al torrente discursivo.

13. Transformadores: producen cambios cualitativos en las unidades básicas dentro del discurso.

14. Correctores: son los encargados de corregir la actividad (misión), efectividad y dirección de las unidades básicas dentro del sistema.

15. Atractor extraño: aquel que incentiva el fin de la situación discursiva y el discurso en cuanto sistema.
Visto esto, se ofrece a continuación el modelo formal. Las barras verticales que dividen el modelo se denominan generaciones. Ellas delimitan los periodos más significativos dentro del modelo y sirven para resaltar (de ser conveniente) las transmutaciones y replicaciones de una misma idea dentro de un mismo y diferentes espacios de tiempo.

Se resaltan, además, cómo en la representación anterior los inputs y outputs del sistema pueden aparecer en cualquiera de los segmentos del modelo. Pero como es natural este solo es un modelo vacío. El objetivo aquí es dejar claro formalmente la estructura posible de un discurso, las variables que lo componen y las relaciones entre ellas.

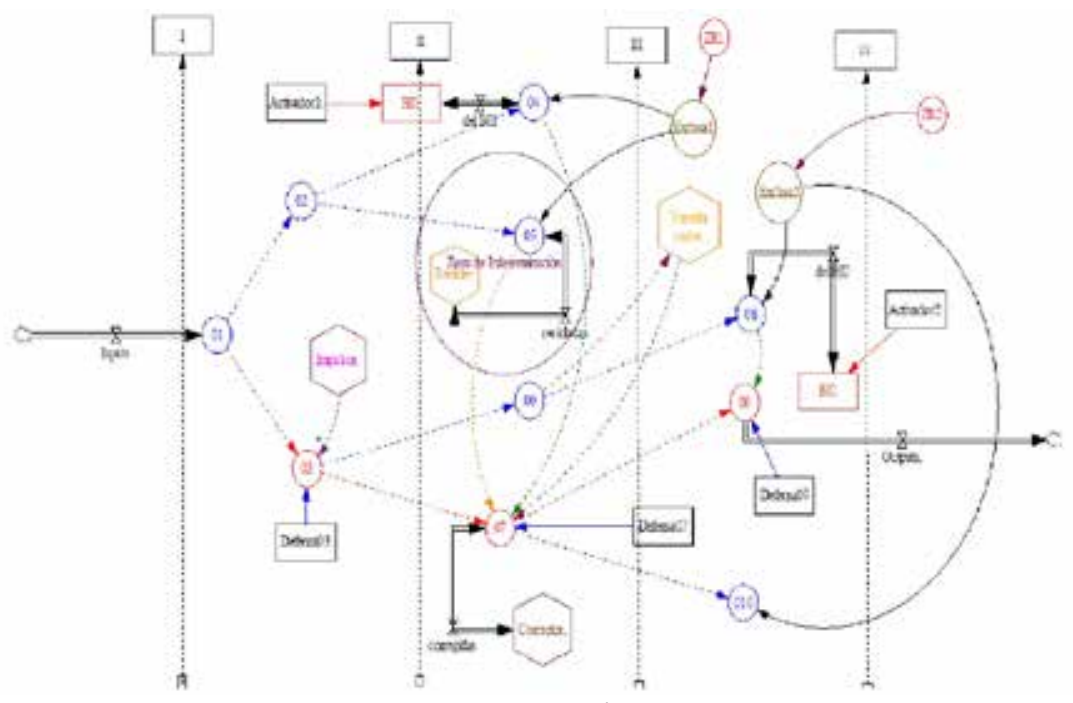

Figura 2. Modelo formal 
Si bien todo lo dicho hasta ahora es teóricamente posible, reconocemos que faltaría, no obstante, aplicarlo a un caso concreto para poder demostrar sus potenciales reales. De ahí que nos propongamos las siguientes consideraciones generales.

\section{Discusión}

Este trabajo trata de llevar el estudio del discurso, especialmente su versión crítica, al cambio de paradigma que se lleva a cabo en las últimas décadas en ciencias sociales. Es una crítica a la crítica del análisis del discurso, en la que se le incorporan nuevos elementos del pensamiento científico proveniente de diferentes campos de estudio y se efectúa el respectivo cambio de noción en la concepción tanto del objeto de estudio como de la forma de abordarlo.

La implementación práctica en una investigación ayudará a complementar y descubrir qué otros elementos deben integrar la teoría del análisis complejo del discurso. Sin embargo, esta, más que un ejercicio de validación (lo cual es científicamente inadmisible, en cuanto un caso no valida la regla), es un ejercicio de reflexión para completar la parte teórica expuesta, lo que viene a tono con los postulados de la "tercera vía" que nace del modelo de simulación basado en agentes.

La utilización de la modelación para analizar sistemas discursivos permite jugar y experimentar con variables y flujos, una vez construido el modelo, para verificar y contrastar posibles variantes en el proceso de análisis. Esto hace el trabajo mucho más fiable, debido a que se puede verificar con esquemas, gráficos, operaciones lógicas, herramientas y derivados del programa las informaciones y los análisis que se desarrollaban en los diferentes niveles de la investigación. Esto posibilita, a su vez, el manejo de grandes volúmenes de datos y no entorpecer la claridad de los resultados obtenidos y reducir el tiempo mínimo indispensable.

\section{Referencias bibliográficas}

Alexander, M. (2009). We do complexity too! Sociology, chaos theory and complexity science. The Future of Sociology.

Byrne, D. S. (1998). Complexity theory and the social sciences: an introduction. Psychology Press.

Bushev, А. В. (2013). Стереотипное в дискурсе глобальных медиа. 
Recuperado de http://www.engectver. ru/Konferenciya/8s/Bushev.php

Carrasco, I. y Vivanco, M. (2011). ¿Sistemas dinámicos en ciencias sociales? Revista de Sociología, 11(26), 169-191.

Dijk, T. van (s. f.). El estudio del discurso (Documento en versión digital disponible en la biblioteca de la Facultad de Comunicación de la Universidad de La Habana).

Dijk, T. van (1994). Discurso, poder y cognición social. Cuadernos de la Maestría en Lingüística, 2.

Dijk, T. van (1996). Análisis del discurso ideológico. México: UNAM.

Dijk, T. van (1997). What is political discourse analysis. Belgian Journal of Linguistics, 11(1), 11-52.

Dijk, T. van (2004). Discurso y dominación. En Grandes conferencias en la Facultad de Ciencias Humanas, 4, 5-28.

Dijk, T. van (2005). Ideología y análisis del discurso. Utopía y Praxis Latinoamericana, 10(29).

Dijk, T. van y Mendizábal, I. R. (1999). Análisis del discurso social y político. Quito: Editorial Abya Yala.
Egüez, P. (1999). Ideología, una aproximación multidisciplinaria. Barcelona: Gedisa.

García, R. (1994). Interdisciplinariedad y sistemas complejos. Ciencias sociales y formación ambiental, 85-124. Golovina, (2008). Experiencia del análisis del discurso: procedimiento capitular de la instrucción municipal en los medios de comunicación regionales. Recuperado de http:// www.training.com.ua/trainer/golovina_ljudmila

Holland, J. (1998). Sistemas adaptativos y complejos. Redes de Neuronas, 259-295.

Howarth, D. (s. f.). Teoría del discurso. Recuperado de http://www.cholonautas.edu.pe

Izquierdo, L. R., Ordax, J. M. G., Santos, J. I. y Martínez, R. D. O. (2008). Modelado de sistemas complejos mediante simulación basada en agentes y mediante dinámica de sistemas. Empiria. Revista de metodología de ciencias sociales, 16, 85-112.

Kuhn, T. S. (2011). La estructura de las revoluciones científicas. Fondo de cultura económica. 
Lara, A. (2008). Sistemas complejos adaptables y teoría de la empresa: el programa de investigación. Economía Informa, 352, 65-92.

Martín García, J. (2003). Teoría y ejercicios prácticos de dinámica de sistemas. Editado por el autor.

Miceli, J., Guerrero, S., Quinteros, R. A., Díaz, D., Kristoff, M. J. y Castro, M. (2005). Teorías de la complejidad y el caos en ciencias Sociales. Modelos basados en agentes y sociedades artificiales. En Actas del I Congreso Latinoamericano de Antropología. Rosario, Argentina: Universidad Nacional de Rosario. Recuperado de http://revista-redes.rediris.es/webredes/arsrosario/06-Miceli\%20y\%20 otros.pdf

Morgenstern, O. y Neumann, J. von (1947). Theory of games and economic behavior. Princenton: Princeton University Press.

Morin, E. (1990). Introducción al pensamiento complejo. La Habana: Félix Varela.

Morin, E. (1992). El paradigma perdido: ensayo de bioantropología. Editorial Kairós.
Morin, E. (1977). El método (vol. I). Madrid: Cátedra.

Ponce Muñoz, P. (2009). Análisis de la teoría de sistemas complejos y su aplicación a sistemas organizacionales. Revismar, 1, 52-67.

Prigogine, I. (1999). Filosofía de la inestabilidad. Voprosy Filosofii, 6, 465.

Ramírez, G., Alonso, M. de L. (2013). Filosofía de la ciencia II. La Habana: Félix Varela.

Santander, P. (2011). Por qué y cómo hacer análisis de discurso. Cinta de moebio, 41, 207-224.

Timoschuk, E. А. (2010). Теория дискурса и межкультурная коммуникация. Recuperado de http:// www.my-luni.ru/journal/3/

Todorov, T. (1990). Genres in discourse. Cambridge University Press.

Thompson, J. B. (2008). Ideología y cultura moderna. La Habana: Pablo de la Torriente Brau y Félix Varela. 